$\Phi=-1$

\title{
A Survey on Diagnosis of US Image Thyroid Nodules and Automated Classification
}

\author{
Mohsin Khan A ${ }^{1}$, Anuj Jain ${ }^{2}$ \\ ${ }^{1}$ Research Scholar, ${ }^{2}$ Research Guide, ${ }^{1,2}$ ECE Dept., Bhagwant University, Ajmer, India. \\ *Corresponding Author Email: ${ }^{\text {}}$ mkjakster@gmail.com, ${ }^{2}$ a1978jain@gmail.com
}

\begin{abstract}
Different types of human diseases are detected by medical image analysis which plays an important role. Studies that are developed for automated thyroid cancer classification is reviewed in this paper, especially to analyze the benign and malignant thyroid nodules features and comparisons. Hypothyroidism, hyperthyroidism, goitre and thyroid nodules (benign/malignant) are thyroid disorders. Ultrasound imaging, CT, MR imaging, nuclear medicine (NM) with positron emission tomography (PET), single photon emission computed tomography (SPECT) are the different medical techniques used to identify and classify thyroid gland abnormalities. In order to enhance the diagnosis of thyroid disease, various image processing techniques applied to thyroid ultra sound images are reviewed here. Studies based on non-clinical features for thyroid nodules classification is also discussed and reviewed.
\end{abstract}

Keywords: Thyroid disorders, Image processing, segmentation, classification, performance measures, Automated Diagnosis.

\section{Introduction}

Ultrasonography and computer assisted tomography are used for diagnosing diseases using imaging techniques such as thyroid nodules which is very common in fifty percent of the adults, among which five percent turns to be malignant. US imaging are economical, advanced and easy to use. Due to the cost effectiveness and portability US imaging are often adopted in hospitals. Thyroid is excellently suitable to ultrasonography for the reason of its echoginity, peripheral position size [1]. Thyroid sector of the neck and alter in voice due to the repetitive laryngeal nerve involvement. The main classifications of thyroid nodule tests are benign and malignant, colloid nodules, benign cysts, macro follicular adenoma and multi nodular goiter are benign lesions. Medical procedure of the nodule is not necessary in this case unless the nodule becomes bigger and causes some problems [2].Early detection of cancer in beginning stage can be easily diagnosed before cancer spreads and death rate is less. FineNeedle Aspiration Biopsy (FNAB), Computed Tomography (CT), MR Imaging (MRI), US imaging, elastography and pathologic studies are the common techniques to detect thyroid nodules. $\mathrm{NAB}$ is the aureate standard for finding malignancy in thyroid nodule [3]. The inceptive objective of the test is to find the presence of nodule in thyroid. The next step is to check whether the found nodule is malignant or benign. Detecting the thyroid cancer at an early stage can reduce the possibility of death rate [4]. The benign and malignant thyroid nodules have distinct ultrasonographic characteristics. Benign nodules exhibit small internal flow when compared to malignant nodules [5]. Malignant nodules have an incidental ring which can be present are absent in benign nodule [5]. High resolution ultra sound and contrast enhanced ultra sound are the two most used US imaging techniques. The thyroid gland is existed as an butterfly structure body consisting of two conoid lobules which belongs to the system of endocrine.
The temperature of the human body and childhood growth and intelligence is regulated by thyroid gland yielding thyroid hormone. The main organ in the body that can absorb iodine is the thyroid gland. Maintaining the body to use energy stay hot and keep the brain, heart muscles and other organ working is done by thyroid hormones. The nodules like hypothyroidism; hyperthyroidism, goiter, benign and malignant etc. are the thyroid disorders caused by abnormalities in thyroid gland [6]. Existence of the nodule present in thyroid may or may not be cancerous. Ultrasonography helps to find there qualities because of the difference in the characteristics of benign and malignant nodule shown in table.1. [1]

Table 1: Benign/Malignant characteristics [1]

\begin{tabular}{|l|l|}
\hline Malignant characteristics & Benign characteristics \\
\hline Irregularity in borders & Smooth border \\
\hline Hypo echoic & Hyper echoic \\
\hline Micro calcifications & Macro calcifications \\
\hline Infiltrative margins & No Infiltrative Margins \\
\hline $\begin{array}{l}\text { Abnormal cervical Lymph nodes } \\
\text { present }\end{array}$ & $\begin{array}{l}\text { Abnormal cervical Lymph nodes } \\
\text { absent }\end{array}$ \\
\hline Increased intra-nodular vascularity & Periphery nodular vascularity \\
\hline
\end{tabular}

The flow diagram of a computer aided design system for thyroid nodule classification is as shown in figure 1 .

The accessible dataset is divided in to training and testing sets.Several characteristics are taken from the training images during the feature extraction phase in the offline system (sonographic/statistical/both). The characteristics that are distinctive and non redundant in information are selected and then fed to the classifiers during the feature selection step. By using the ground truth and selected features classifiers are trained to check the image is benign or malignant.

Several test images are evaluated and class brands are equated with the ground truth of the test images. Classifier performance measure like precision, sensitivity, specificity and PPV are 
calculated. The classifier with best precision is selected as the optimal classifier for implementation in future.

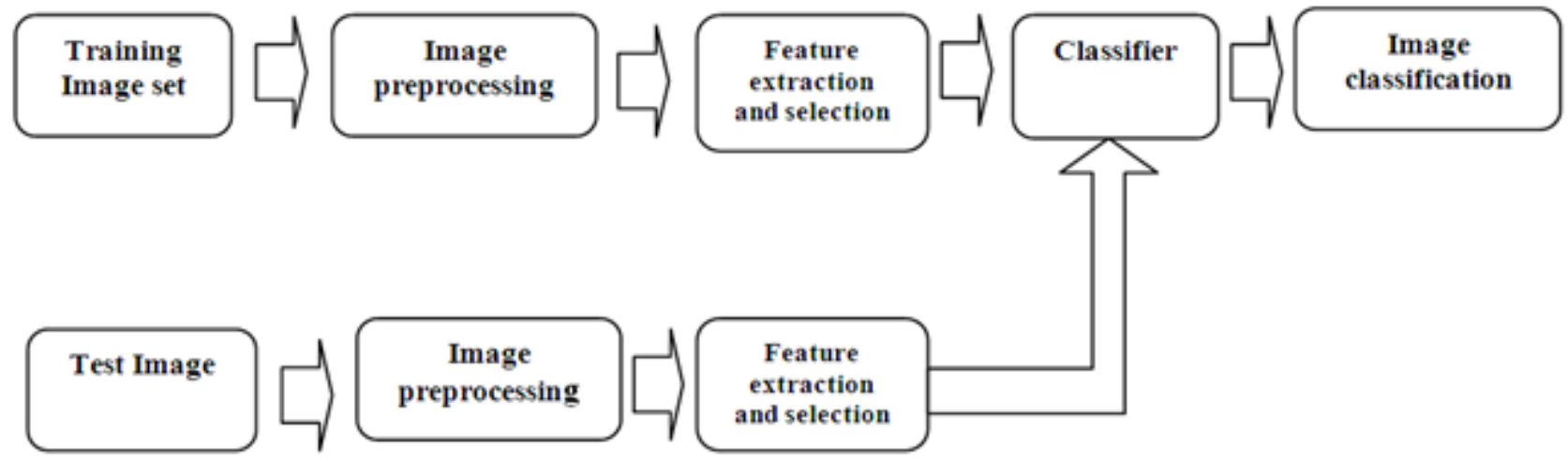

Fig. 1: Flow diagram for automated thyroid nodule classification

\section{Ultrasound Image Data Features and Acquisition}

\section{Types of benign Lesion}

The images for thyroid nodule benign US is as shown in figure 2.(A) Cystic (B) Hypo echogenic nodules (C) Echogenic nodules. Cystic: Cystic is a benign lesion of around $3 \mathrm{~cm}$ diameter. Density of fluid causes lack of homogeneity.

Nodule 1: An oval shape small benign lesion with longitudinal axis of around $1.5 \mathrm{~cm}$ is called nodule 1 having clear margin which is hypo echogenic with calcifications and lacks vascularity.

Nodule 2: These hypo echogenic lesions are very deceitful.

Nodule 3a and 3b: Nodules of size $1.5 \times 3 \times 2 \mathrm{~cm}$ diameter which is $\mathrm{T}$ hypo echogenic with poor homogeneity, vascularity, clear margins, lacks halo and calcifications.

Nodule 4: This is similar to nodule 3 but vascularity is inside the nodule, with size around $1 \times 3 \times 1.5 \mathrm{~cm}$.

Nodule 5: An echogenic nodule with fine margins which is a big benign with lack of halo and calcifications. Vascularity very poor.

Nodule 6: In this the size is $3 \times 3 \times 4 \mathrm{~cm}$, fine margin, with fluid degeneration features with lack of halo and calcification. Vascularity very poor.

Nodule 7: A non homogeniciso-normogenic area with $1.5 \mathrm{~cm}$ diameter which is degraded with lack of vascularity and blurs margins.
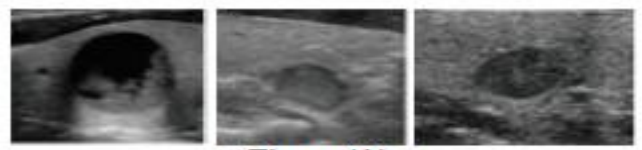

Figure (A)
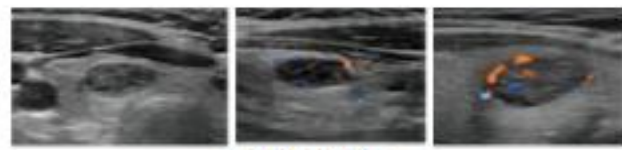

Figure (B)
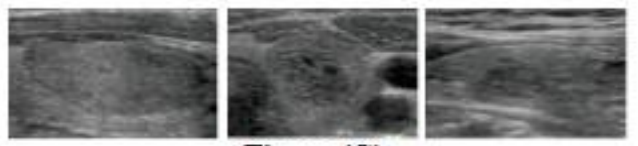

Figure (C)

Fig. 2: US images of benign thyroid nodule: (A) cystic

(B) hypo echogenic nodules (C) is echogenic nodules

\section{Types of malignant lesions}

Malignant US images of thyroid nodule shown in figure 3.

Thyroid cancer 1a and 1b: An hypoechogenic, having size of $2 \times 3 \times 2 \times 3 \times 3 \mathrm{~cm}$, malignant lesion with poor homogeneity, blur margins,untypical shape with axis transversal to a main axis of thyroid lobe, having calcification and deep hypoechogenic areas with higher lesion vascularity.

Thyroid cancer 2: Type of the malignant lesion which is histological papillary thyroid cancer and not similar to cancerous nodules beside the echogenicity. Hypoechogenic nodule of size $1.5 \times 3 \times 2 \times 3 \times 1 \mathrm{~cm}$, having partially blur margins could be taken for malignant with oval shape and longitudinalaxis, short fall of vascular detection and no specific micro calcifications.

Thyroid cancer 3 and 4:A papillary thyroid cancer of size $3 \times 3 \times 2 \times 3 \times 2 \mathrm{~cm}$ nodule with not such typical US image features. The typical features of cancer are hypo echogenicity, poor homogeneity with internal fluid area, vascularity with arterial flow of high values $\mathrm{V}_{\max } 40 \mathrm{~cm} / \mathrm{s}$. The other untypical characteristics are having clear margin with full halo, shape of oval without any micro calcifications. The obtained US images equivalent to different variant indicates the challenges in visual diagnosis of benign and malignant thyroid nodules. Interpretations of visual inspection are extremely subjective due to subtle changes in the image features. With a proper choice of feature extraction and classification procedures US images can be made as a reliable tool for diagnosis of thyroid nodule.
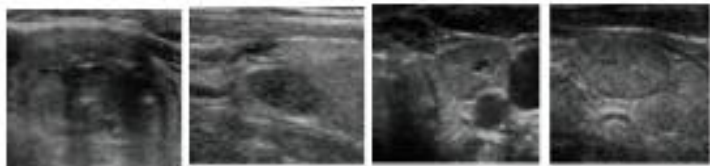

Fig. 3: Malignant US images of thyroid nodule

\section{Extraction of Features}

Sonographic Features: To detect malignancy in thyroid nodules these features are used that an endocrinologist observes in US images. An explanation of these features and the corresponding categories is shown below [7].

Internal content: It can be defined as the ratio of solid and cystic portions present in nodule mass. The nodules can be grouped into five categories depending upon this parameter as shown in table 2.

Table 2: Categories of thyroid nodule based on internal content [7]

\begin{tabular}{|l|l|}
\hline Category of nodule solid & Description of the category \\
\hline Predominantly solid & $90 \%$ of the nodule is solid \\
\hline Predominantly cystic & The nodule is more than $50 \%$ solid \\
\hline Cystic & The nodule is more than $50 \%$ cystic \\
\hline Spongiform & $\begin{array}{l}\text { More than } 50 \% \text { of the nodule consists } \\
\text { of small and uniform cysts, thus nodule } \\
\text { look like a sponge. }\end{array}$ \\
\hline
\end{tabular}

Shape: Categories on shape are taller than wide, ovoid to roundand irregular.

Echogenicity: The classification of thyroid nodule based on echogenecity is done as hypo echoic, is echoic and hyper echoic. Markedly hypo echoic whose echogenicity of nodule is less than 
the strap muscles. Hypo echoic is having less echogenecity than surrounding thyroid parenchyma, which is bigger than the adjacent muscle. Is echoic implies thatechogenicity is same as the thyroid, while hyper echoic echogenicity is greater than the thyroid.

Calcifications: This can be grouped as micro calcification, macro calcification rim calcifications and nil calcifications. In micro calcification the size of the calcium deposit is around $1 \mathrm{~mm}$ diameter or less, where as in macro calcification it is $1 \mathrm{~mm}$ and above. Rim calcification is defined as the presence of calcification around the nodule.

Echo Texture: The two types of echo texture are homogeneous and heterogeneous.

Margin: The types of margin can be well-defined margin, illdefined margin and well-defined smooth and speculated.

Capsule invasion: Happened or nor happened.

Halo: Present or absent.

Radiologists classify the thyroid nodules as likely benign, sceptical for malignancy and undetermined. The possible features of malignancy can be micro calcification, micro lobulated border, marked hypoechogenicity and taller than wide based on sonographic features [8].

\section{Textural Features}

The examples of textural features can be smoothness, coarseness and pixel regularity. Textural analysis can be done either by structural or by statistical methods, statistical analysis methods like entropy, homogeneity, symmetry energy and contrast are more suitable for image analysis and they are less complex [9]. A brief explanation about different texture based features is given below.

Gary Level Co-occurrence Matrix (GLCM) texture features: The distribution of co occurring values at a given offset over an image is defined as GLCM. GLCM measures the equality between two pixels that are apart, extracted from the homogeneity feature. The entropy feature will have highest value when all the elements of the co occurrence matrix are same.

Fractal Dimension (FD): Based on the concept of fractal geometry the FD concept is started. It can be defined as the irregularity of an image or surface roughness. Since the echo graphic patterns gives marked distinctness in the benign and malignant images of thyroid nodules FD is very helpful for thyroid cancer diagnosis [10].

Local Binary Pattern (LBP): The mixture of structural and statistical characteristics of an image is given by LBP which is developed by Ojalaet al. [11],

Fourier Spectrum Descriptor (FSD): Changes in the shape of an image is quantified by FSD.

Laws Texture Energy (LTE): Texture energy can be measured by LTE. By applying the energy texture transforms to the image the estimation of energy within the past region of the filter can be done [12].

\section{Classification}

To classify a particular class input observation is learnt by classifiers. Supervised learning is preferred for classification task. The class label of benign/malignant are put to assist the classifier trained during the learning step. Unknown test patterns are fed after learning. Based on the knowledge got during the learning step, the system will classify the class of the unknown image. The most commonly used classifiers which are used in detection of thyroid nodule is summarised below.

Gaussian Mixture Model (GMM): The basis functions for Gaussian functions which are merged to provide multimodal density in GMMis a generalised basic network functions. For tuning the GMM and for its performance evaluation using the test data is done by using expectation-maximization algorithm during the training phase of the GMM [13].

Support Vector Machine (SVM): A set of hyper planes in a high dimensional space or a hyperplane or exists in SVM. Hyper planes are used for classification. The selection of hyper plane is done such that it forms great separation among the two classes. In particular the separation from the hyper plane to the convenient sample or each side is increased. Samples belonging to different are divided by very clear and large gap. Samples as points in a space done by SVM mapping [14].

K-Nearest Neighbour (KNN): The classification of unknown samples which is done by associating the unknown to known sample based on some distance or a some criterion is called KNN classifier which is an instance based classifier [15]. Here a class is assigned to a sample which is common in k-nearest neighbours with a small positive number for $\mathrm{k}$ set of neighbours are taken as training samples to the classifier to know the correct classification. Probabilistic Neural Network (PNN): A multilayered feed forward network organised as four layers is operated as PNN. The four layers can be input layer, pattern layer, summation layer and output layer. A two layered radial basis network which can be used for classification purpose is RBPNN [16]. Evaluation of the distance vector by approximating the distance between input and the training input vector is done at the first layer. The summation of contributions for each input classes and yielding a probability vector at its output is done by the next layer.The maximum probability of the test data can be used by the complete transfer function to evaluate the unknown class.

Decision Tree (DT) classifier: A DT classifier, in which the separation of training set is done in a recursive way, with each division containing superior samples from one class. A complicated decision making process is splitted into measurable decisions and the output is generated as a tree like structure in binary using DT classifier. The rules that are used to classify the unknown class are derived from the various classes which are evaluated from tree [17].

Adaboost classifier: To improve the performance of the weak classifiers a Meta classifiers used called adaboost classifier. The machine learning algorithm of this classifier which relates the input training set to a exhausted learner algorithm frequently. The algorithm conserves and updates a set of weights for the training set in every repetations. The beginning of the algorithm is done by keeping all the weights to be of similar value. The weights are updated after each call by increasing weights of incorrectly classified examples.

Navies Bayes Classifier (NBC): Based on bayes theorem the NBC classifier is designed which assumes that existence of specific characteristics of a class is not related or not dependent on the existence of any other characteristics. Estimation of parameter is done by using Navies Bayed model which uses the technique of maximum likelihood [19].

Fuzzy Sugeno Classifier: Using subtractive clustering, Fuzzy inference system is generated .A feature space is generated during the phase of training by using a set of fuzzy rules consisted by FIS. Calculation of fuzzy inference are performed to test the class of text data [20].Classifier efficiency is done by calculating the features namely accuracy, explicitly and acuteness. Detection of thyroid nodule is done by the classifier parameters having higher capability and reliability.

\section{Results and Limitations}

Detection of thyroid malignancy is analyzed by suitable features taken out of US images which is a non-invasive and affordable. Features of US images for detection of thyroid malignancy have been used by many studies. Observations are made that the features of taller than broad shape, marked hypo echogenicity, micro and macro calcifications, micro lobulated or margin irregularity, enlarged blood flow in nodule by Doppler indication, 
solid pattern and vascularity of intranodule local invasion, regional lymphadenopathy and existence of numerous nodules firmly specifies the malignancy[28, 21-24].Cystic and spongiform and cystic nodules are predominantly haves more calcification with diameter greater than $1 \mathrm{~mm}$ or rim like calcification are benign in nature generally[8]. Hyper echoic or Isohypo nodules with shape irregularity or an oval shape to round having a margin which can be expertly defined or poorly defined and presence of rim calcification is classified as indefinite [25-27]. Number of studies proved that sonographic characteristics are well used for detection of thyroid malignancy. The occurrence of inter and intra observer variability in illumination of result is the main limitation of this analysis which is experienced even by medical proficient.Park et al. [7] initiated that the inter-observer affiliation was modest for the shape characteristics, calcification and echogenicity, while the affiliation was good for the margin characteristics, echo texture and invasion of capsules. The values $65.3 \%-81.9 \%, 60.7 \%-68.9 \%, 69.7 \%-73.8 \%$, and $66.6 \%-75.5 \%$ are average range of sensitivity, specificity, positive predictive value, and negative predictive values respectively. Hong et al. [8] observed that the values $38.6 \%, 28.2 \%, 49.4 \%$ and $59.8 \%$ are predicted positive values of malignancy which are found by using sonographic characteristics of micro calcifications, micro lobulated margin, marked hypoechogenicity of shape taller than wide.

\section{Conclusion}

Medical images interpretation, accommodating a reliable alternate assessment in finding lesions, tumours and highlighting their locations, evaluating disease seriousness and leads to more exact diagnosis resolution with improved computerised analysis. It can be proved that automated thyroid nodule diagnosis systems are more objective, fast and accurate. A brief review about some of the sonographic and non-clinical characteristics and most commonly used classifiers for classification of thyroid nodules is presented in this paper.

\section{References}

[1] Sheeja Agustin A, S.SureshBabu, "Thyroid segmentation on US images: An Overview", International Journal of Emerging Technology and Advanced Engineering, Vol.02, Issue 12, pp 8893, (Feb 2013).

[2] Slough, C. M., Randolph, G. W. Workup of well-differentiated thyroid carcinoma. Cancer Control 13, 99-105 (2006).

[3] Baloch, Z. W., Fleisher, S., LiVolsi, V. A., Gupta, P. K. Diagnosis of "follicular neoplasm": a gray zone in thyroid fine-needle aspiration cytology. DiagnCytopathol 26, 41-44 (2002). DOI: 10.1002/ dc. 10043

[4] Baskin, H. J., Duick, D. S. The endocrinologists' view of ultrasound guidelines for fine needle aspiration. Thyroid 16, 207 208 (2006). DOI:10.1089/thy.2006.16.207

[5] Ivanac, G., Brkljacic, B., Ivanac, K., Huzjan, R., Skreb, F., Cikara, I. Vascularisation of benign and malignant thyroid nodules: CD US evaluation. Ultraschall Med 28, 502-506 (2007).

[6] JeetendraGochare, PallaviChoudhary, "Pixel measurement of thyroid gland by using ultrasound images", International Journal of Engineering Sciences and Research Technology, Vol.05, Issue 7 , pp. 883-888, (July 2016).

[7] Park, C. S., Kim, S. H., Jung, S. L., Kang, B. J., Kim, J. Y., Choi, J. J., Sung, M. S., Yim, H. W., Jeong, S. H. Observer variability in the sonographic evaluation of thyroid nodules. J Clin Ultrasound 38, 287-293 (2010). DOI: $10.1002 /$ jcu. 20689

[8] Hong, Y. J., Son, E. J., Kim, E. K., Kwak, J. Y., Hong, S. W. Chang, H. S. Positive predictive values of sonographic features of solid thyroid nodule. Clin Imaging 34, 127-133 (2010). DOI: 10.1016/ j.clinimag.2008.10.034

[9] Acharya, U. R., Faust, O., Sree, S. V., Molinari, F., Garberoglio, R. Suri, J. S. Cost-effective and non-invasive automated benign and malignant thyroid lesion classification in 3D contrast-enhanced ultrasound using combination of wavelets and textures: A class of thyroscan algorithms. Technol Cancer Res Treat 10, 371-380 (2011)

[10] Acharya, U. R., VinithaSree, S., Krishnan, M. M., Molinari, F., Garberoglio, R., Suri, J. S. Non-invasive automated 3D thyroid lesion classification in ultrasound: a class of thyroscan systems. Ultrasonics 52, 508-520 (2012). DOI: 10.1016/j.ultras.2011.11.003

[11] Ojala, T., Pietikainen, M., Maenpaa, T. Multiresolutiongray-scale and rotation invariant texture classification with local binary patterns. IEEE Trans Pattern Anal Mach Intell 24, 971-987 (2002). DOI: 10.1109/TPAMI.2002.1017623

[12] Laws, K. I. Rapid Texture Identification. Image Processing for Missile Guidance. San Diego, Society of Photo-Optical Instrumentation Engineers (1980).

[13] Bilmes, J. A. A gentle tutorial of the EM algorithm and its application to parameter estimation for Gaussian Mixture and Hidden Markov Models. International Computer Science Institute 1-13 (1998)

[14] Vapnik, V. Statistical learning theory (Adaptive and Learning Systems for Signal Processing, Communications and Control Series). John Wiley \& Sons, New York (1998).

[15] Larose, D. T. KNN. In: Discovering Knowledge in Data: An Introduction to Data Mining, 1st Ed., pp. 90-106. New Jersey; Wiley Interscience (2004).

[16] Specht, D. F. Probabilistic neural networks. J Neural Networks 3 , 109-118 (1990). DOI: 10.1016/0893-6080(90)90049-Q

[17] Larose, D. T. Decision Trees. In: Discovering Knowledge in Data: An Introduction to Data Mining, 1st Ed., pp. 108-126. New Jersey; Wiley Interscience (2004).

[18] Freund, Y., Schapire, R. E. A decision-theoretic generalization of on-line learning and an application to boosting. J Comp SystSci 55, 119-139 (1997). DOI: 10.1006/jcss.1997.1504

[19] Han, J., Kamber, M. Data Mining: Concepts and Techniques. Morgan Kaufmann (2006).

[20] Sugeno, M. Industrial Applications of fuzzy Control. Elsevier Science, New York (1985)

[21] Cappelli, C., Castellano, M., Pirola, I., Cumetti, D., Agosti, B., Gandossi, E., AgabitiRosei, E. The predictive value of ultrasound findings in the management of thyroid nodules. QJM 100, 29-35 (2007). DOI: 10.1093/qjmed/hcl121

[22] Chan, B. K., Desser, T. S., McDougall, I. R., Weigel, R. J., Jeffrey, R. B. Jr. Common and uncommon sonographic features of papillary thyroid carcinoma. J Ultrasound Med 22, 1083-1090 (2003).

[23] Khoo, M. L., Asa, S. L., Witterick, I. J., Freeman, J. L. Thyroid calcification and its association with thyroid carcinoma. Head Neck 24, 651-655 (2002).

[24] Papini, E., Guglielmi, R., Bianchini, A., Crescenzi, A., Taccogna, S., Nardi, F., Panunzi, C., Rinaldi, R., Toscano, V., Pacella, C. M Risk of malignancy in nonpalpable thyroid nodules: Predictive value of ultrasound and color-doppler features. J ClinEndocrinolMetab 87, 1941-1946 (2002).

[25] Algin, O., Algin, E., Gokalp, G., Ocakoglu, G., Erdogan, C., Saraydaroglu, O., Tuncel, E. Role of duplex power doppler ultrasound in differentiation between malignant and benign thyroid nodules. Korean J Radiol 11, 594-602 (2010). DOI: 10.3348/ kjr.2010.11.6.594

[26] Jang, M., Kim, S. M., Lyou, C. Y., Choi, B. S., Choi, S. I., Kim, J. $\mathrm{H}$. Differentiating benign from malignant thyroid nodules: Comparison of 2- and 3-dimensional sonography. J Ultrasound Med 31, 197-204 (2012)

[27] Rago, T., Di Coscio, G., Basolo, F., Scutari, M., Elisei, R., Berti, P., Miccoli, P., Romani, R., Faviana, P., Pinchera, A., Vitti, P. Combined clinical, thyroid ultrasound and cytological features help to predict thyroid malignancy in follicular and hupsilonrthle cell thyroid lesions: Results from a series of 505 consecutive patients. ClinEndocrinol (Oxf) 66, 13-20 (2007). DOI: 10.1111/j.13652265.2006.02677.x

[28] Gul, K., Ersoy, R., Dirikoc, A., Korukluoglu, B., Ersoy, P. E., Aydin, R., Ugras, S. N., Belenli, O. K., Cakir, B. Ultrasonographic evaluation of thyroid nodules: comparison of ultrasonographic, cytological, and histopathological findings. Endocrine 36, 464-472 (2009). DOI: 10.1007/s12020-009-9262-3 